\title{
Nuclear Medicine and Resources for Patients: How Complex Are Online Patient Educational Materials?
}

\author{
David R. Hansberry ${ }^{1}$, Kush Shah ${ }^{2}$, Nitin Agarwal $^{3}$, Sung M. Kim ${ }^{1}$, and Charles M. Intenzo ${ }^{1}$ \\ ${ }^{1}$ Department of Radiology, Thomas Jefferson University Hospital, Philadelphia, Pennsylvania; ${ }^{2}$ Department of Medicine, Rowan \\ School of Osteopathic Medicine, Stratford, New Jersey; and ${ }^{3}$ Department of Neurological Surgery, University of Pittsburgh Medical \\ Center, Pittsburgh, Pennsylvania
}

\begin{abstract}
The Internet is a major source of health care information for patients. The American Medical Association and the National Institutes of Health recommend that consumer health care websites be written at a third- to seventh-grade level. The purpose of this study was to evaluate the level of readability of patient education websites pertaining to nuclear medicine. Methods: We searched for 10 terms on Google, collected the top 10 links for each term, and analyzed their level of readability using 10 well-established readability scales. Results: Collectively, the 99 articles were written at a grade level of 11.8 (SD, 3.4). Only 5 of the 99 articles were written at the third- to seventhgrade level recommended by the National Institutes of Health and the American Medical Association. Conclusion: There is a clear discordance between the readability level of nuclear medicinerelated imaging terms and the National Institutes of Health and American Medical Association guidelines. This discordance may have a negative impact on patient understanding, contributing to poor health outcomes.
\end{abstract}

Key Words: nuclear medicine; readability; health literacy; patient education

J Nucl Med Technol 2018; 46:144-146

DOI: $10.2967 /$ jnmt.117.203380

$\mathbf{T}$ oday, the Internet serves as an enormous source of medical information, especially for patients. It contains valuable resources, is easily accessible from almost anywhere, and helps patients make decisions, for better or for worse, pertaining to their health. In fact, the number of individuals using the web to access health information rose from 54 million in 1998 to over 117 million in 2005 (1). Over $80 \%$ of adults research health issues on the Internet, yet more than half fail to communicate this newfound information to their physician $(2,3)$, thus placing a great deal of responsibility on these websites regarding their readability. Readability describes how easily a consumer

\footnotetext{
Received Oct. 7, 2017; revision accepted Dec. 6, 2017.

For correspondence or reprints contact: David R. Hansberry, Department of Radiology, Thomas Jefferson University Hospitals, 132 S. 10th St., Philadelphia, PA 19107.

E-mail: david.hansberry@jefferson.edu

Published online Feb. 2, 2018.

COPYRIGHT (c) 2018 by the Society of Nuclear Medicine and Molecular Imaging.
}

can comprehend written text, taking into account content, style, format, and organization. In a study by the National Assessment of Adult Literacy, 36\% of Americans had no more than basic health literacy (the ability to understand simple health information to make appropriate health care decisions), and another 5\% were not even competent enough to participate in the survey $(4,5)$. These data suggest that over 90 million Americans struggle to understand the content of the websites they use to obtain their health care information.

The National Institutes of Health and the American Medical Association recommend that patient education resources be written at a third- to seventh-grade level $(6,7)$. However, recent studies have shown that this recommendation is not always followed. It appears that many medically relevant web sources overestimate the reading level of their users. Hansberry et al. reviewed the readability of patient education resources from RadiologyInfo.Org and found that the articles were written at a 10th- to 14th-grade level (8). One area of medicine in which readability is particularly important is nuclear medicine, because it is vital for patients to understand any procedure they may undergo to ensure its success and prognostic value.

Given the complexity of nuclear medicine and the general public's likely interest in learning about it, we used several standard assessment scales to evaluate the level of readability of nuclear medicine-related websites written for the lay public.

\section{MATERIALS AND METHODS}

In November 2016, a Google search was performed using 10 search terms, and the top 10 links for each term were collected and individually analyzed for their level of readability using 10 wellestablished quantitative readability scales. The search terms included nuclear imaging, nuclear medicine, PET scan, cardiac stress test, thyroid scan, VQ scan, bone scan, gamma ray, dexa [dual-energy x-ray absorptiometry] scan, and radiotracers. Websites not written exclusively for patients were excluded from the analysis. One of the articles, on radiotracers, contained too few words to be analyzed with the 10 readability scales and was excluded from the analysis. The quantitative readability scales were the Coleman-Liau Index, Flesch-Kincaid Grade Level, Flesch Reading Ease, FORCAST (Ford, Caylor, Sticht) Formula, Fry Graph, Gunning Fog Index, New Dale-Chall, New 


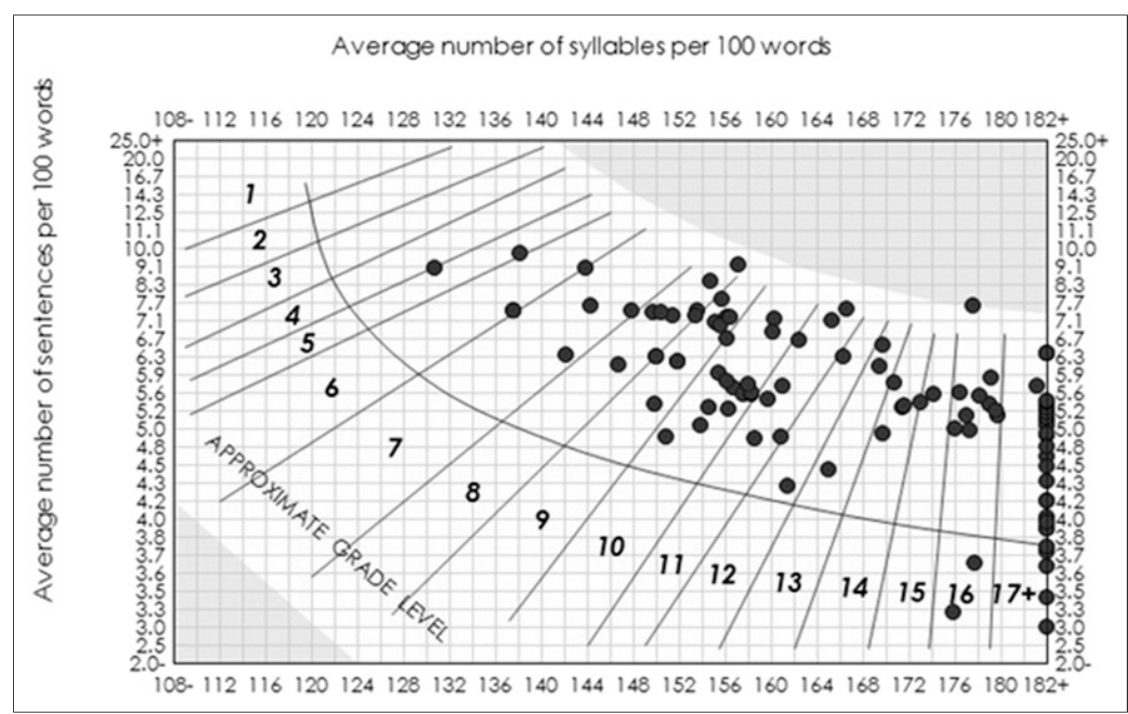

FIGURE 1. Fry Graph with circles correlating with individual articles and their respective levels of readability.

Fog Count, Raygor Readability Estimate, and Simple Measure of Gobbledygook.

The Coleman-Liau Index measures how understandable text is using characters (9). Both the Flesch-Kincaid Grade Level and the Flesch Reading Ease gauge how challenging text is but emphasize different factors $(10,11)$ : the Flesch-Kincaid Grade Level places greater weight on sentence length than on word length, whereas the Flesch Reading Ease does not. The Flesch Reading Ease creates a score of $0-100$, with lower scores indicating more difficult text. The FORCAST Formula analyzes only vocabulary, making this scale helpful for text without proper structure (12). The Fry Graph calculates the grade reading level by considering the average number of sentences and syllables and displays it on a graph (13). The Gunning Fog Index estimates the education level needed for a person to understand a passage on the first reading

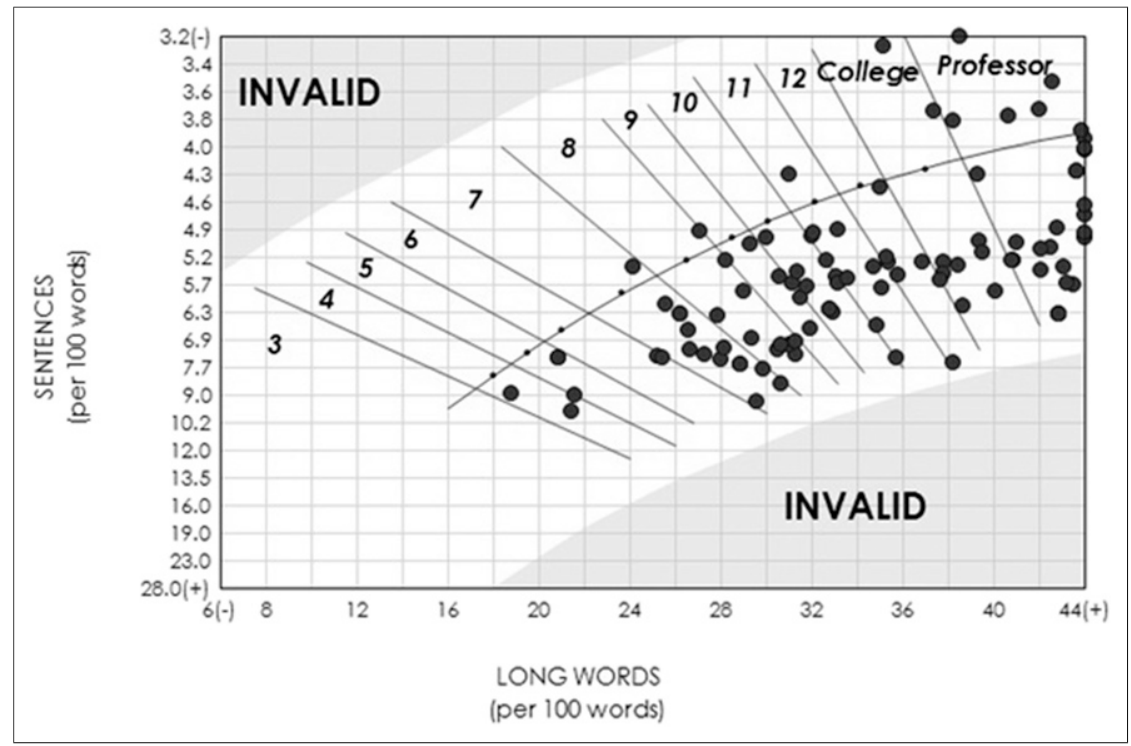

FIGURE 2. Raygor Readability Estimate with circles correlating with individual articles and their respective levels of readability.
(14). The New Dale-Chall calculates how difficult it is for readers to comprehend text comprising words that most Americans can understand (15). The New Fog Count is adapted from the Gunning Fog Index and considers sentence length and words containing 3 or more syllables to calculate grade level (11). The Raygor Readability Estimate evaluates the average number of sentences and letters per hundred words and plots them graphically to estimate grade level (16). Lastly, the Simple Measure of Gobbledygook determines the number of years of education required to understand a passage (17).

\section{RESULTS}

In total, 99 articles were analyzed. The Coleman-Liau Index found the level of readability to be at a grade level of 11.4 (SD, 2.7). The Flesch-Kincaid Grade Level reported the average readability to be at a grade level of 11.6 (SD, 3.6). The Flesch Reading Ease reported a mean score of 45.9 out of 100 (SD, 18.5) for the comfort with which a patient can understand these materials. The FORCAST Formula and New Dale-Chall determined the grade level required to understand the articles to be 10.8 (SD, 1.1) and 11.5 (SD, 3.2), respectively. Similarly, both the Fry Graph (Fig. 1) and the Raygor Readability Estimate (Fig. 2) found that the average grade level-12.9 (SD, 3.9) and $11.6(\mathrm{SD}, 4.1)$, respectively - was too high for the average American. The Gunning Fog Index found the readability to be at a grade level of 13.2 (SD, 3.3), whereas the Simple Measure of Gobbledygook reported a grade level of 13.6 (SD, 2.8). The New Fog Count discovered the average readability to be at a grade level of 9.9 (SD, 3.2), which was the lowest but still much higher than recommended by the National Institutes of Health and the American Medical Association. Collectively, the 99 articles were written at a grade level of 11.8 (SD, 3.4). Only 5 of the articles were written at the third- to seventh-grade level recommended by the National Institutes of Health and the American Medical Association. Additionally, to be fully understood, $47 \%$ of the material required at least a high school education ( $>12$ th grade) and $11 \%$ required at least a college education ( $>16$ th grade).

\section{DISCUSSION}

The power of the Internet as a primary source of medical information is stronger now than ever as patients use it to build their medical knowledge 
and influence their health care decisions. Over $80 \%$ of all adults use web sources to look up health information, with the possibility of accessing a seemingly endless number of medically related texts and web pages (2). Yet despite this shift in primary medical resources, there appears to be a disconnection between the readability of medical websites and the literacy level of the average American. In fact, numerous studies evaluating the reading level of patient education resources among a variety of specialties, including interventional radiology and neuroradiology, have found them to be written far above the average American's literacy level $(18,19)$.

With regard to nuclear medicine, the Internet can serve a vital role in helping patients further understand the field and clarify any misconceptions. However, the results of this study show that there is a clear discrepancy between the reading level at which nuclear medicine resources are written and the reading level of the patients these resources are meant to inform. The implications of this chasm range from patients who are deterred from potentially essential procedures to patients who develop complications due to noncompliance. Therefore, given the data presented in this study and the consequences, it seems efficacious to reevaluate how nuclear medicine resources are written. If such resources are easier to understand, patients may seek nuclear medicine testing more often.

The limitations of this study include those inherent in readability scales. They do not take into consideration the understandability of a word. For example, although DEXA (dual-energy x-ray absorptiometry) and gamma are short words, the average patient is unlikely to fully understand what they mean, and although polysyllabic words such as appointment are reported by the scales as being more complex, some of these words may be understood by a wide audience. Additionally, readability scales do not factor in word order, which affects patient understanding. We also did not evaluate videos, images, or other nontextual information that can contribute to how the written information is interpreted. Another limitation concerns the homogeneity in Internet use by patients with different levels of health literacy. It is possible that less literate people are less likely to use the Internet. Therefore, future research may include investing in other avenues to spread comprehensible information to all patients regardless of their literacy level. It may also be beneficial to evaluate each individual search term to see whether some terms have a lower-grade reading level than others (e.g., the term bone scan may be deemed more readable than PET scan). Altogether, these improvements may help alleviate some of the limitations of the study.

\section{CONCLUSION}

The Internet is an omnipresent source of medical information. The results of this study show that websites intended to educate and inform patients about nuclear medicine are written at a reading level well above that of the average American. This finding may stimulate a reassessment of how nuclear medicine material is expressed and whether it should be altered to include simpler sentences with smaller words to reach a broader population of patients. Such changes may greatly aid both patients and physicians.

\section{DISCLOSURE}

No potential conflict of interest relevant to this article was reported.

\section{REFERENCES}

1. The Harris Poll. The Harris Poll website. www.harrisinteractive.com/news/newsletters/healthnews/HI_HealthCareNews2005Vol5_Iss08.pdf. Published July 28, 2005. Accessed January 5, 2017.

2. Fox S, Rainie L. The online health care revolution: how the web helps Americans take better care of themselves. Washington, DC: Pew Internet and American Life Project; 2000.

3. Bylund CL, Gueguen JA, Sabee CM, Imes RS, Li Y, Sanford AA. Providerpatient dialogue about Internet health information: an exploration of strategies to improve the provider-patient relationship. Patient Educ Couns. 2007;66: 346-352.

4. Kutner M, Greenburg E, Jin Y, Paulsen C. The Health Literacy of America's Adults: Results from the 2003 National Assessment of Adult Literacy. Washington, DC: National Center for Education Statistics; 2006. NCES 2006-483.

5. Weiss BD. Health Literacy and Patient Safety: Help Patients UnderstandManual for Clinicians. Chicago, IL: American Medical Association Foundation; 2007.

6. Weis BD. Health Literacy: A Manual for Clinicians. Chicago, IL: American Medical Association Foundation and American Medical Association; 2003.

7. How to write easy-to-read health materials. National Library of Medicine website. https://medlineplus.gov/etr.html. Updated June 28, 2017. Accessed March 6, 2018.

8. Hansberry DR, John A, John E, Agarwal N, Gonzales SF, Baker SR. A critical review of the readability of online patient education resources from RadiologyInfo. Org. AJR. 2014;202:566-575.

9. Coleman M, Liau TL. A computer readability formula designed for machine scoring. J Appl Psychol. 1975;60:283-284.

10. Flesch R. A new readability yardstick. J Appl Psychol. 1948;32:221-233.

11. Kincaid JP, Fishburne RP Jr, Rogers RL, Chissom BS. Derivation of New Readability Formulas (Automated Readability Index, Fog Count and Flesch Reading Ease Formula) for Navy Enlisted Personnel. Springfield, VA: National Technical Information Service; 1975.

12. Caylor JS, Sticht TG, Fox LC, Ford JP. Methodologies for Determining Reading Requirements of Military Occupational Specialties. Alexandria, VA: Human Resources Research Organization; 1973.

13. Fry E. A readability formula that saves time. J Reading. 1968;11:513-516, 575578.

14. Gunning R. The Technique of Clear Writing. New York, NY: McGraw-Hill; 1952.

15. Chall JS, Dale E. Readability Revisited: The New Dale-Chall Readability Formula. Northampton, MA: Brookline Books; 1995.

16. Raygor AL. The Raygor readability estimate: a quick and easy way to determine difficulty. In: Pearson PD, ed. Reading: Theory, Research, and Practice. 1977: 259-263.

17. McLaughlin GH. SMOG grading: a new readability formula. J Reading. 1969;12: 639-646.

18. Hansberry DR, Kraus C, Agarwal N, Baker SR, Gonzales SF. Health literacy in vascular and interventional radiology: a comparative analysis of online patient education resources. Cardiovasc Intervent Radiol. 2014;37:1034-1040.

19. Hansberry DR, Agarwal N, Gonzales S, Baker S. Are we effectively informing patients? A quantitative analysis of on-line patient education resources from the American Society of Neuroradiology. Am J Neuroradiol. 2014;35:12701275 . 